OPEN ACCESS

Edited by:

Paulette Van Vliet,

University of Newcastle, Australia

Reviewed by:

Fabien Chauveau, INSERM U1028 Centre de Recherche en Neurosciences de Lyon, France Jose Gutierrez,

Columbia University, United States

*Correspondence:

Thiago L. Russo

thiagoluizrusso@gmail.com

Specialty section: This article was submitted to

Stroke,

a section of the journal

Frontiers in Neurology

Received: 04 February 2018

Accepted: 16 July 2018

Published: 28 August 2018

Citation:

Alcantara CC, García-Salazar LF,

Silva-Couto MA, Santos GL,

Reisman DS and Russo TL (2018)

Post-stroke BDNF Concentration Changes Following Physical Exercise:

A Systematic Review.

Front. Neurol. 9:637.

doi: 10.3389/fneur.2018.00637

\section{Post-stroke BDNF Concentration Changes Following Physical Exercise: A Systematic Review}

\author{
Carolina C. Alcantara ${ }^{1}$, Luisa F. García-Salazar ${ }^{1,2}$, Marcela A. Silva-Couto ${ }^{1}$, \\ Gabriela L. Santos ${ }^{1}$, Darcy S. Reisman ${ }^{3}$ and Thiago L. Russo ${ }^{1 *}$ \\ ${ }^{1}$ Laboratory of Neurological Physiotherapy Research, Physical Therapy Department, Federal University of São Carlos, São \\ Carlos, Brazil, ${ }^{2}$ Escuela de Medicina y Ciencias de la Salud, Gl Ciencias de la Rehabilitación, Universidad del Rosario, \\ Bogotá, Colombia, ${ }^{3}$ Department of Physical Therapy, University of Delaware, Newark, DE, United States
}

Background: Research over the last two decades has highlighted the critical role of Brain-derived neurotrophic factor (BDNF) in brain neuroplasticity. Studies suggest that physical exercise may have a positive impact on the release of BDNF and therefore, brain plasticity. These results in animal and human studies have potential implications for the recovery from damage to the brain and for interventions that aim to facilitate neuroplasticity and, therefore, the rehabilitation process.

Purpose: The aim of this study was to carry out a systematic review of the literature investigating how aerobic exercises and functional task training influence BDNF concentrations post-stroke in humans and animal models.

Data Sources: Searches were conducted in PubMed (via National Library of Medicine), SCOPUS (Elsevier), CINAHL with Full Text (EBSCO), MEDLINE 1946-present with daily updates (Ovid) and Cochrane.

Study Selection: All of the database searches were limited to the period from January, 2004 to May, 2017.

Data Extraction: Two reviewers extracted study details and data. The methodological quality of the studies that used animal models was assessed using the ARRIVE Guidelines, and the study that evaluated human BDNF was assessed using the PEDro Scale.

Data Synthesis: Twenty-one articles were included in this review. BDNF measurements were performed systemically (serum/plasma) or locally (central nervous system). Only one study evaluated human BDNF concentrations following physical exercise, while 20 studies were experimental studies using a stroke model in animals. A wide variation was observed in the training protocol between studies, although treadmill walking was the most common type of intervention among the studies. Studies were of variable quality: the studies that used animal models scored from $8 / 20$ to $15 / 20$ according to the ARRIVE Guidelines. The only study that evaluated human subjects scored 5/10 according to the PEDro scale and, which indicates a quality classified as "fair".

Conclusions: The results of the current systematic review suggest that aerobic exercise promotes changes in central BDNF concentrations post-stroke. On the other 
hand, BDNF responses following functional exercises, such as reaching training and Constraint Induced Movement Therapy (CIMT), seem to be still controversial. Given the lack of studies evaluating post-stroke BDNF concentration following physical exercise in humans, these conclusions are based on animal work.

Keywords: Brain-derived neurotrophic factor, neuroplasticity, exercise, rehabilitation, stroke

\section{INTRODUCTION}

Research over the last 10 years has demonstrated that Brainderived neurotrophic factor (BDNF) plays an important role in brain plasticity in the intact brain $(1,2)$, as well as after central nervous system (CNS) damage $(3,4)$. BDNF is a member of the neurotrophin family, known for its role in neuronal proliferation, survival and differentiation (1). Along with its receptor tyrosine kinase, this neurotrophin is largely distributed throughout the healthy human brain $(5,6)$.

The role of BDNF after stroke has been highlighted in many studies $(3,4,7-10)$. Its action is related not only to the induction of anti-apoptotic mechanisms, reducing the size of the lesion, but also to secondary neuronal death (3) Furthermore, motor learning post-stroke has been related to increases in BDNF concentrations in the cortex (10), which may accomplish cortical map reorganization through synaptogenesis, enhanced dendritic spine formation and ramification, thereby contributing in many ways to neuronal plasticity post-stroke $(3,4,9)$.

Given the evidence linking BDNF and brain plasticity, research advancements have been made aiming to understand the response of BDNF levels to physical exercise training and how these changes would mediate the beneficial effects of exercise on learning $(8,11,12)$. This systematic review specifically emphasized studies that examined exercise intervention, not exercise as a priming (e.g.,-single session, before and after exercise measurements only). Converging results available in the literature suggest that aerobic exercise training may lead to an increase in BDNF concentrations in neurologically intact humans (13). Thus, using aerobic training as an intervention to optimize neuroplasticity and recovery in patients post-stroke has gained considerable interest (10). Although there is still a lack of studies evaluating BDNF concentrations following exercise in subjects post-stroke, evidence based on strokeinduced animal models suggests a relationship between aerobic exercise training and an increase in BDNF concentrations (1416) Furthermore, recent studies have also measured BDNF concentrations following functional task training in strokeinduced animals models, such as skilled reach training, in order to further clarify the mechanisms by which these interventions would induce recovery $(17,18)$.

However, given the controversial results between studies, the relationship between exercise training (aerobic and/or functional task training) and BDNF levels post-stroke has not been fully elucidated. Although previous literature reviews have addressed the effects of aerobic exercise on neuroplasticity in general after stroke $(7,8)$ the focus of the current review is on the role of BDNF in the physical exercise response, either aerobic and/or functional task training, in human or animal models of stroke. Thus, the primary objective of this study was to carry out a systematic review of literature investigating the effects of aerobic exercise and functional task training on BDNF concentrations in animals or humans post-stroke. The secondary objective of this review was to analyze the methodological quality of selected studies.

\section{METHODS}

\section{Search Methods}

Searches were conducted in May 2017 using the following electronic databases: PubMed (via National Library of Medicine), SCOPUS (Elsevier), CINAHL with Full Text (EBSCO), MEDLINE 1946-present with daily updates (Ovid) and Cochrane. The following MeSH headings or keywords were used: "Brain Derived Neurotrophic Factor" OR "BDNF" AND "stroke" OR "cerebrovascular accident". All of the database searches were limited to the period from January, 2004 to May, 2017.

For the search and study selection, the following inclusion criteria were used: (1) the primary objective of the study was to evaluate the effect of physical exercise (aerobic exercise and/or functional task training) on BDNF concentrations in humans or animals and (2) full papers published in English. Studies were excluded in the following sequence: any article that did not involve BDNF; studies that evaluated BDNF levels in other conditions, rather than stroke; studies that had primary objectives other than stated in \#1 above (e.g.,-validation of an analysis method); and interventions that did not involve physical exercise. Furthermore, reviews, case studies, commentaries, letters and guidelines were excluded.

\section{Study Selection}

To identify potentially eligible articles, two reviewers (CCA and LFG) independently assessed the titles and abstracts obtained from the electronic search according to inclusion and exclusion criteria. After this first selection, full-length articles were read by the same reviewers in order to define which articles met all the inclusion criteria. However, if there was a disagreement between two reviewers, a third reviewer was consulted. If the full text was not available, the authors were contacted and asked if they could send their research papers. Furthermore, the authors verified the reference lists from each included paper to check if there were relevant publications (not included in the initial search) and manually searched for them. To systematize and organize the search and data extraction, the State of the Art through Systematic Review (StArt) (Available from: http://lapes.dc.ufscar.br/tools/ start_tool) was used. 


\section{Data Extraction and Quality Assessment}

A standardized electronic data extraction form was developed to obtain key information relevant to this review. Data extraction for each article were: sample size, characteristics of injury (type-mechanism and time post-injury), species evaluated, measuring technique, site and type of tissue (whether systemically or locally measured, and in which tissue, for example, serum, plasma, brain, muscle, etc.), protocol of exercise, instruments of assessment, time between measurements and main results. The last item (main results) involves the change in BDNF concentrations (increased, decreased or not changed) and if there was any correlation between change concentrations and motor impairment.

The methodological quality of the included studies that used animal models was assessed using the ARRIVE Guidelines. It consists of 20 items, such as the number and specific characteristics of animals used and the experimental, statistical and analytical methods (including details of methods used to reduce bias such as randomization and blinding). The methodological quality of the included study that evaluated human BDNF (19) was assessed using the Physiotherapy Evidence Database (PEDro) Scale (20). The checklist consisted of 11 items related to the study design, reporting eligibility criteria, between-group statistical comparisons and variability measures. In accordance with the total score from the PEDro Scale, the article can be classified into: excellent (10-11), good (6-9), fair (4-5), and poor (less than 4).

\section{RESULTS}

A total of 8744 articles were identified through database searches (Cochrane, $n=1,674$; SCOPUS, $n=954$; CINAHL, $n=778$; PubMed, $n=1,995$; MEDLINE, $n=3,343)$. After the duplicates were removed, 7372 articles were listed, although 3 of them were mentioned as "unclassified articles". Therefore, the titles and abstracts of 7369 articles were screened. Out of these, 7334 articles were excluded primarily because they did not involve BDNF measurements or were evaluated BDNF levels in other conditions, rather than stroke. Finally, 35 full-text manuscripts were read. Another 3 articles from the references were added to the search. In the end, only 21 articles fulfilled all of the inclusion criteria (Figure 1).

Table 1 presents information regarding the included studies that evaluated the effects of physical activity on the BDNF levels. The table is divided into three sections, according to the type of exercise investigated in the study: (1) aerobic exercise; (2) functional training; (3) both (aerobic exercise and functional training). Aerobic exercise (mainly treadmill training) was the most common type of intervention among the studies and most of them showed an increase in BDNF concentration after this intervention. Only one study included in this review was completed in human subjects (19) 0.2 (5). Therefore, most of the understanding of changes in the BDNF concentration with exercise arises from animal studies. Generally speaking, this systematic review showed that central BDNF responses following non-aerobic exercise training in the animal model of stroke are still controversial, whereas aerobic exercise training appears to promote changes in central BDNF concentrations post-experimental stroke in animals.

\section{Methodological Characteristics}

The included studies have a longitudinal design, i.e., BDNF concentrations were assessed after conducting an exercise training program. A wide variation was observed in exercise training protocols between studies regarding duration, intensity and frequency of treatment sessions and the time post-stroke at which the training started (Table 1). Two main types of exercise were addressed in the studies included in this review: (1) Aerobic exercise; and/or (2) Functional training.

\section{Aerobic Exercise}

Fifteen studies evaluated the effect of aerobic exercise training (Treadmill/ Bicycle ergometer/Motorized running wheel) on BDNF concentrations (14-16, 19, 21-31, 35). Only one study evaluated systemic BDNF concentration following aerobic exercise training in humans post-stroke (19), while all others evaluated central BDNF concentration in animal models of stroke. The sample size per experimental group varied from 4 to 20. The methods used to measure the BDNF concentration include ELISA, Immunohistochemistry and Western Blotting. The time at which the BDNF measurement was performed varied from 8 days post-injury until 91 days post-injury among the studies/experimental groups.

\section{Functional Training}

Among all 21 studies included in this review, 6 studies evaluated the effects of functional training on BDNF concentration (17, 18, 32-35) All of them were performed using an experimental stroke model in rats. Functional training such as reaching tasks, Constraint-Induced Movement Therapy (CIMT) and acrobatic training were performed in those studies. One of them compared BDNF concentrations after isolated reaching training to those following a reaching training preceded by aerobic exercise (34). The sample size per experimental group varied from 5 to 12 among these 4 studies. The methods used to measure the BDNF concentration include Immunohistochemistry, Western Blotting and Gene expression (PCR). The time at which the BDNF measurement was performed varied from, 7 days post-injury to 43 days post-injury among the studies/experimental groups.

\section{Methodological Quality of Studies}

All articles that used animal models were scored using the ARRIVE Guidelines. The score ranged from $8 / 20$ to $15 / 20$. All these articles provided, for example, an accurate summary of the background in the Abstract, provided details of the animals used, and described the outcomes and estimation (reported the results for each analysis carried out with a measure of precision). None of them reported regarding adverse effects or described any modifications to the experimental protocols made to reduce adverse events. Besides, none of them explained how the number of animals was decided or provided details of any sample size calculation used. The detailed score for each study in every 


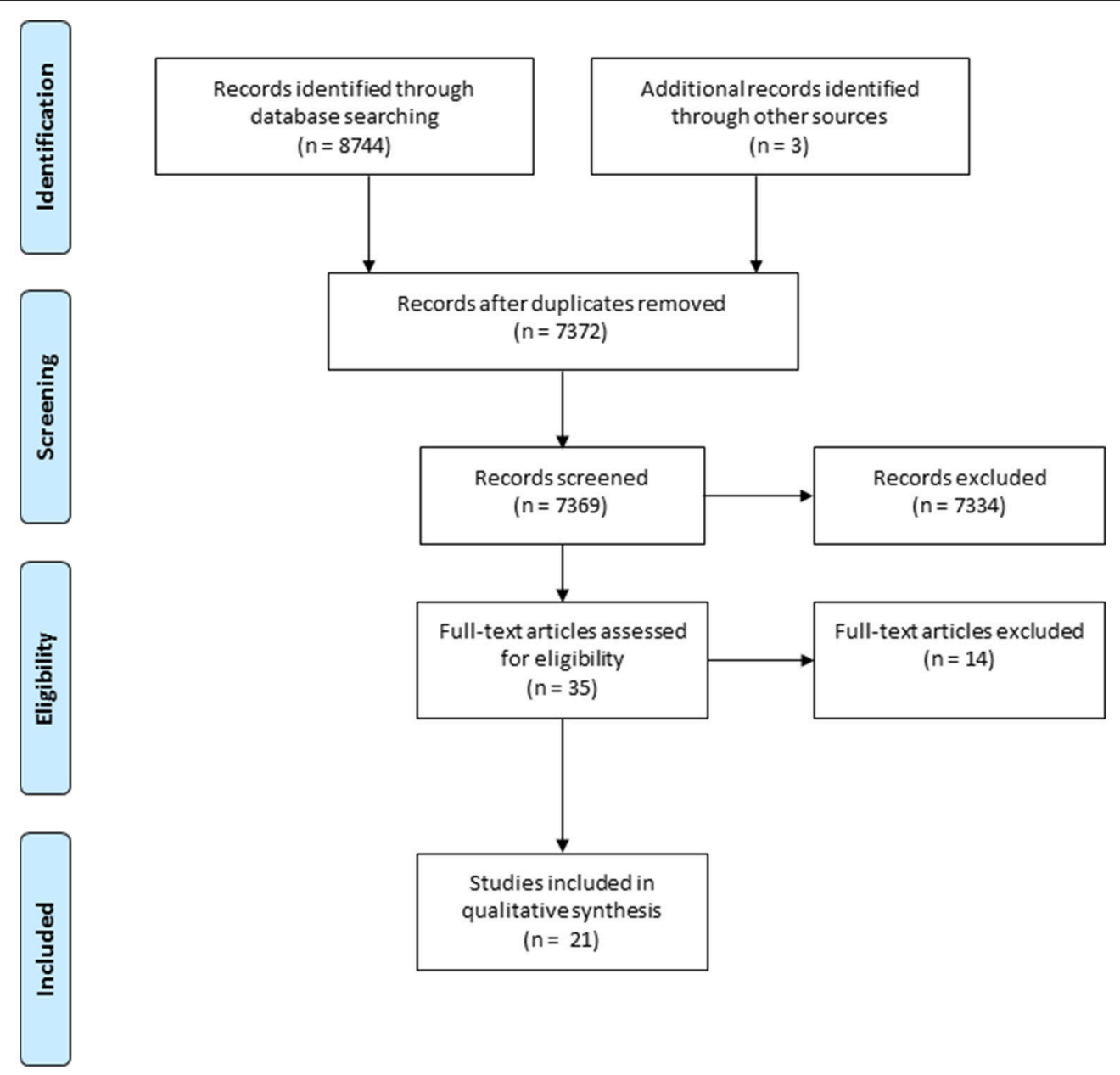

Flow Diagram model From: Moher D, Liberati A, Tetzlaff J, Altman DG, The PRISMA Group (2009). Preferred Reporting Items for Systematic Reviews and Meta-Analyses: The PRISMA Statement. PLoS Med 6(7): e1000097. doi:10.1371/journal.pmed1000097

FIGURE 1 | Flowchart for literature search.

item of the ARRIVE Guidelines is presented as Table 1S. The only study that evaluated human subjects was assessed using the PEDro scale and scored 5/10 according to this scale, which indicates a quality classified as "fair." This study met criteria such as concealed sample allocation, baseline comparability (similar baseline between groups for main outcomes) and blinded subjects.

\section{DISCUSSION}

Recent studies have highlighted BDNF as an important neurotrophic factor involved in motor learning, recovery and neural rehabilitation after a stroke $(7,8,36)$. The current review illustrates the consistencies and discrepancies in the literature regarding the response of $\mathrm{BDNF}$ concentrations following physical exercise training in subjects post-stroke or animals submitted to experimental CNS injury. It is worth mentioning that, although some investigations in humans have been performed recently, much of the understanding of changes in the BDNF concentration with exercise derives from animal studies. For example, only one study included in this review was completed in human subjects (19).

In general terms, aerobic exercise training appears to promote changes in central BDNF concentrations post-experimental stroke in animals, while central BDNF responses following nonaerobic exercise training in the animal model of stroke are still controversial. Most studies included in this review evaluated brain (local) BDNF concentrations in animal experimental designs. Treadmill training was the most common type of intervention among the studies and most of them showed an increase in BDNF concentration after this intervention. On the other hand, a few studies were performed to investigate the effect of non-aerobic training on BDNF concentration, such as reaching training $(32,34)$ and constraint induced movement therapy (CIMT) $(17,18)$, and the results among these studies are in discordance. Besides the differences in intervention protocols, a considerable variety of methods used to measure the BDNF concentrations was observed among the studies. Most of the 


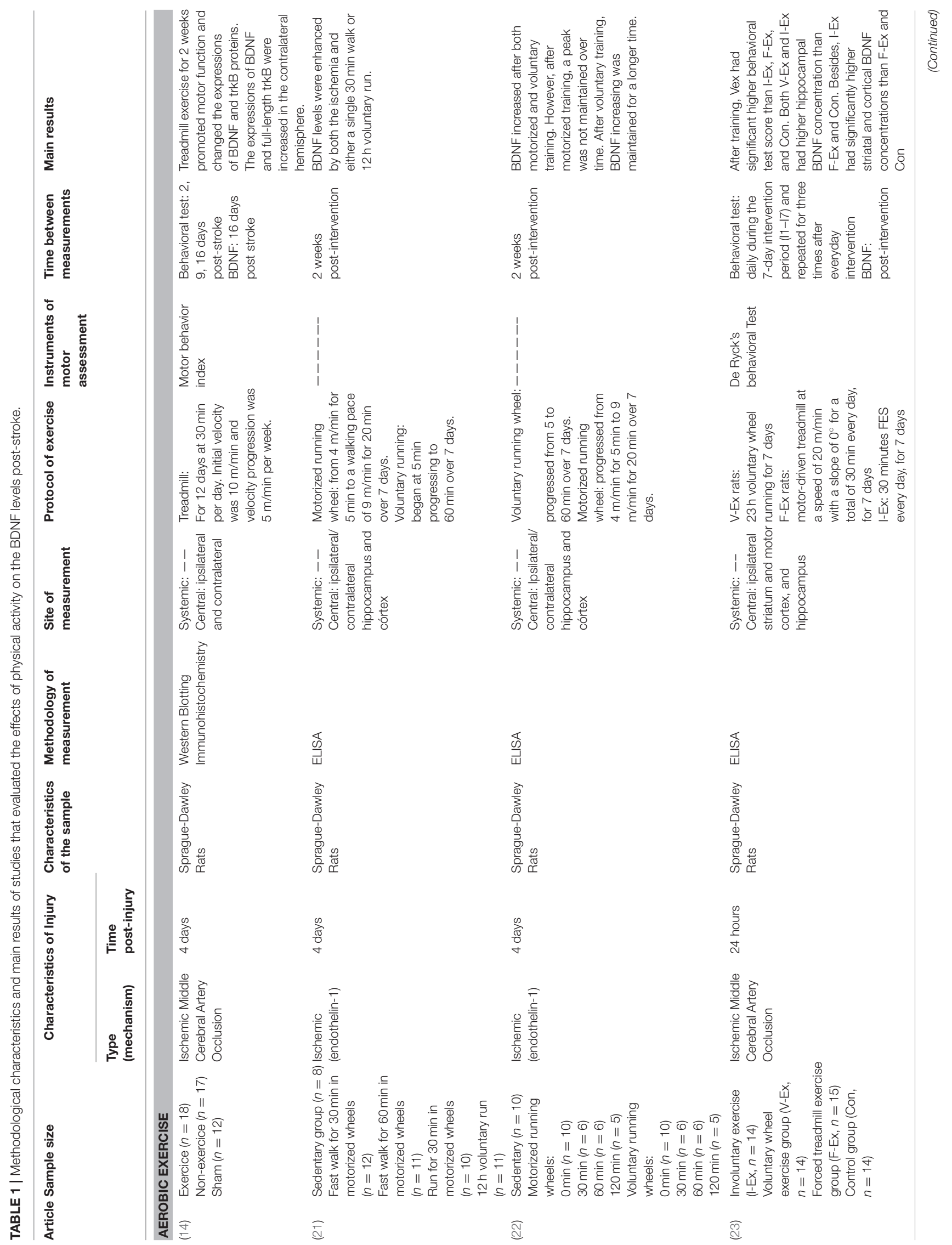




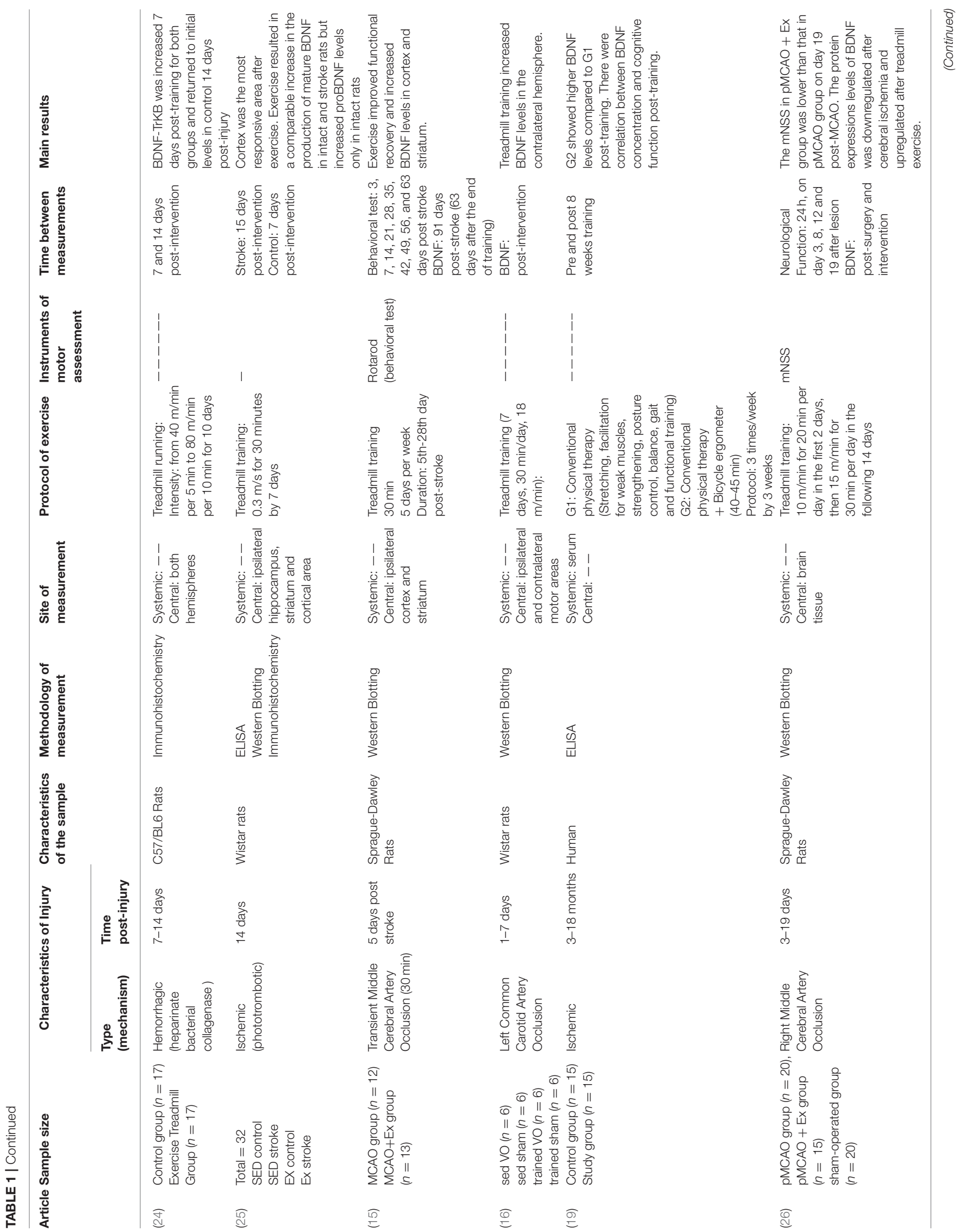




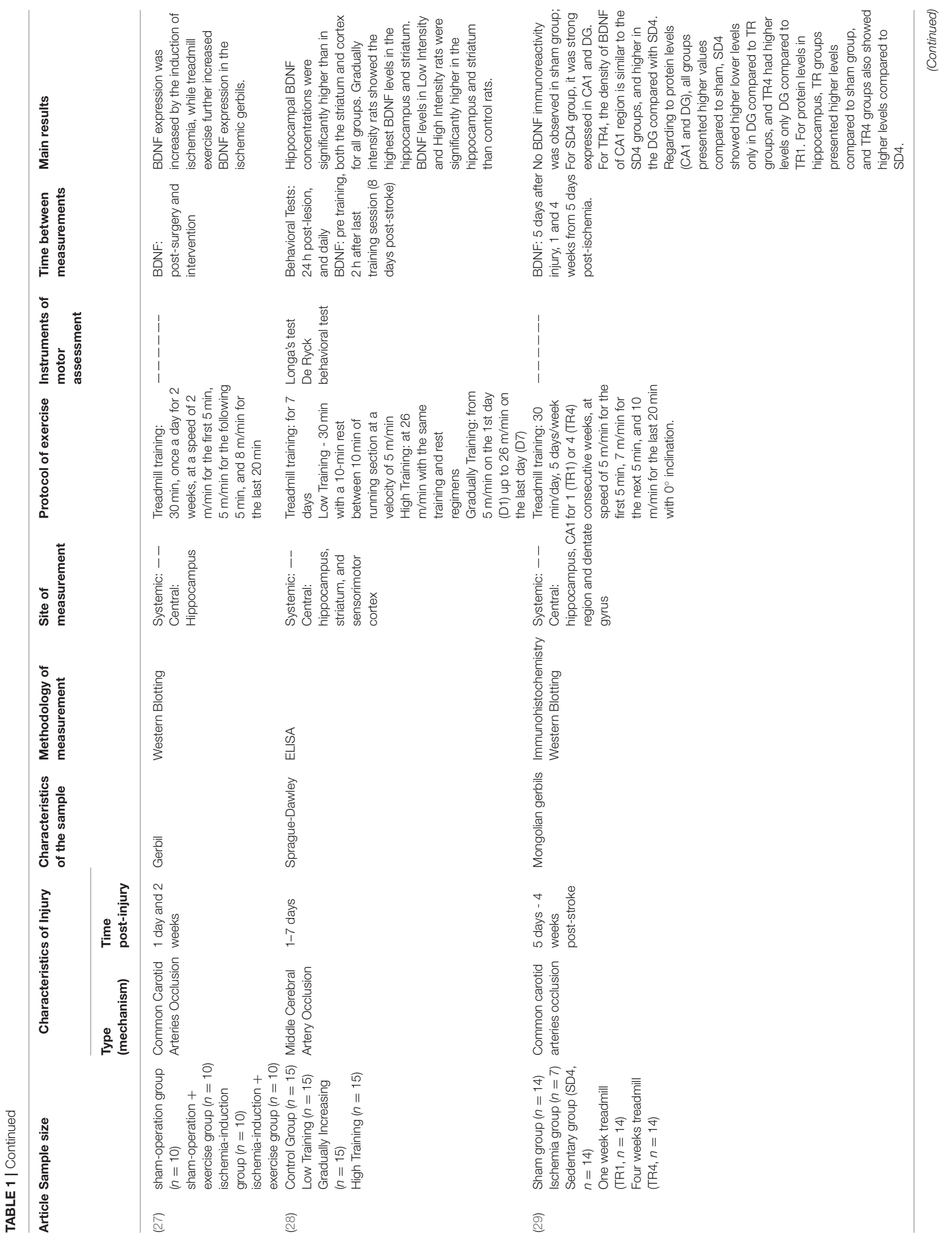




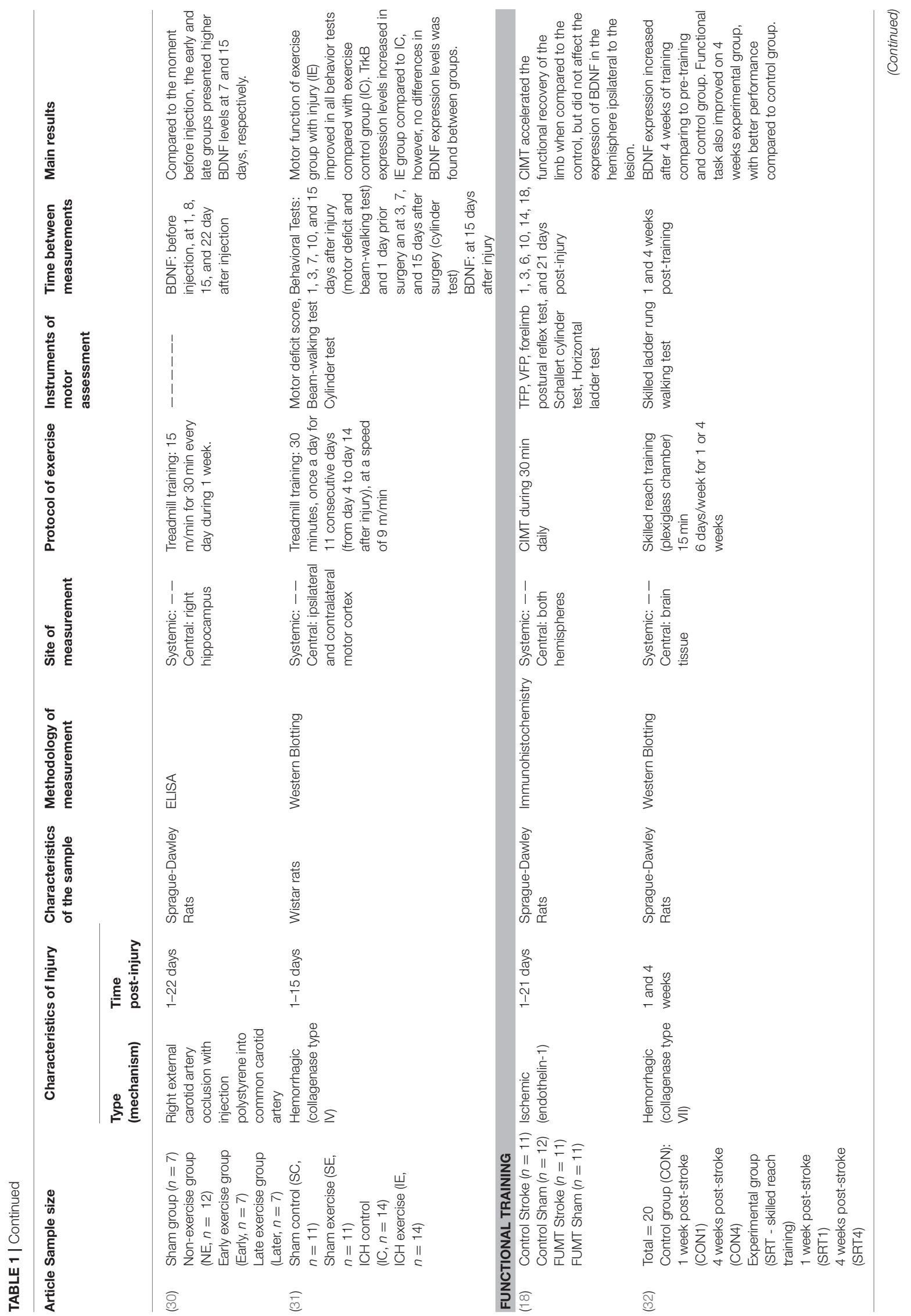




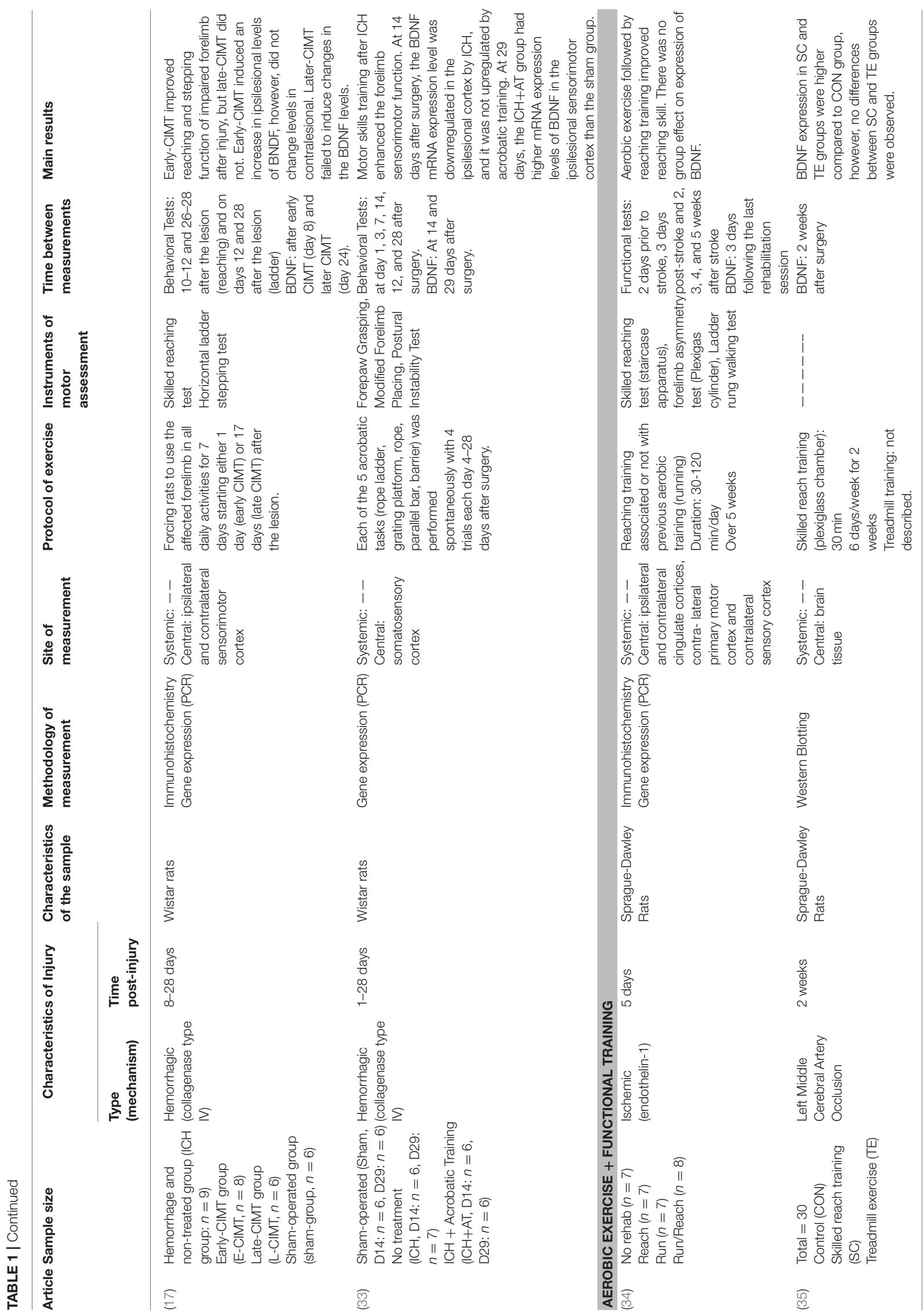


methods used to measure BDNF concentration (e.g., ELISA, PCR, Western Blotting, Immunohistochemistry) are sensitive techniques, although they provide specific information regarding BDNF concentration. For example, ELISA provides an accurate protein quantitation in homogenates, while Western Blotting is not so accurate for quantitation, but provides information about the possible different molecular variants (e.g., pro-BDNF vs. BNDF mature). These differences should be taken into account when comparing the results of the studies.

\section{Aerobic Training}

Aerobic exercise, such as treadmill training, is continually used in rehabilitation due to its effectiveness in improving function, mobility and cardiovascular fitness in patients with chronic stroke (37-39). Generally, this review shows that aerobic exercise is able to promote changes in central BDNF concentrations in animal models of stroke. These results are in accordance with a previous systematic review showing that forced exercise at moderate to high intensity increases BDNF in multiple brain regions in animal models of stroke (8). However, the brain area where these changes occur varied among the studies included in the current review $(14-16,19,21-28)$. Both ipsilateral and/or contralateral changes in BDNF concentrations were found in the studies, and the most common brain areas evaluated were hippocampus, striatum and motor cortex. For example, Quirie et al. (25) evaluated the effect of treadmill training on central BDNF concentrations and, as compared to the striatum and the hippocampus, the cortex showed the greatest increase in BDNF following training. Other authors, however, observed a stronger increase in BDNF concentrations in the hippocampus following treadmill training (28). The different findings might be related to the time after the end of training session at which the BDNF was measured. It seems that when measured immediately after the last treatment session, the hippocampus shows higher BDNF levels. More studies are needed to confirm this hypothesis.

Training parameters such as the type of aerobic exercise, intensity and duration are also important factors that might affect BDNF concentration and motor function recovery $(21-23,28)$. Sun et al. (28) evaluated the effect of different treadmill training intensities on motor function recovery and neurorehabilitation, including the analysis of BDNF levels early after an experimental stroke in an animal model. Briefly, authors identified that training with gradually increased intensity achieved higher BDNF levels and better recovery, although an increase in BDNF was also observed following a low or high intensity exercise (see Table 1 for protocol details). Furthermore, there is also evidence that lower intensity endurance exercise leads to a more prolonged upregulation in central BDNF concentrations (up to $2 \mathrm{~h}$ ) when compared to short periods of intense exercise (22). Such findings suggest that the brain BDNF response may be intensity sensitive, which is in agreement with previous findings from neurologically intact human subjects $(40,41)$. However, conclusions regarding the most effective aerobic training parameters for increasing BDNF concentrations are still limited post-stroke, given the large heterogeneity across studies available in the literature.

Only one study evaluated systemic BDNF concentration after exercise training (19). El-Tamawy et al. (19) investigated the effect of aerobic exercise (bicycle ergometer) on the serum BDNF concentration of post-stroke subjects (19). Although the heterogeneity of the sample was considerable (time post-stroke varying from 3 to 18 months), serum BDNF concentration increased significantly after 8 weeks of bicycle ergometer training, compared to pre-training and compared to the group that was submitted only to conventional physical therapy. Although this study does show relevant information regarding the effect of aerobic exercise training on BDNF concentrations post stroke in humans, the intensity of training was not described in the study. Previous studies in neurologically intact human subjects provide evidence that aerobic exercise training increases BDNF levels and the magnitude of BDNF increase seems to be exercise intensity dependent $(13,40,42)$. Therefore, the training parameters and effort levels should be addressed in future studies in subjects post-stroke to identify the most appropriate training parameters that would result in increased levels of BDNF.

\section{Functional Training}

The findings arising from studies that evaluated the effect of functional training on BDNF levels are controversial. Among six studies, three showed an increase in central BDNF concentration following functional training $(17,32,35)$, while the other three observed no change $(18,33,34)$. A possible reason for this inconsistency might be related to the type of functional training and differences in training parameters. The time post-experimental stroke when the BDNF concentrations were evaluated varied among studies as well, which could be another source of disagreement between results. Studies in neurologically intact humans have also shown discrepancies in the effect of non-aerobic training, such as strength training, on BDNF levels (42-44). Accordingly, it may not be surprising that the results of non-aerobic training studies (functional training) post-stroke and its effects on BDNF concentrations are inconsistent.

In addition to examining the effects of aerobic exercise training on BDNF, studies in neurologically intact subjects have examined the effect of a short bout of aerobic exercise coupled with functional task practice $(45,46)$. These studies suggest that aerobic exercise may in fact "prime" the brain to learn subsequent motor tasks $(45,46)$. Although the mechanisms underlying this "priming" phenomenon are not completely clear, the upregulation of BDNF within the CNS following aerobic exercise could be a contributing factor that facilitates motor learning (10). Among all studies included in the current review, however, only one evaluated the effect of aerobic training (running) performed immediately before a reaching task on functional recovery and BDNF levels poststroke (experimental model). There was a trend for slightly higher levels of central BDNF in both groups that performed an isolated reaching task or running immediately before the reaching task when compared to control, but no significant differences were observed. Therefore, further investigation is needed in order to clarify the role of BDNF in aerobic exercise applied as a "priming" before or after motor learning task poststroke. 


\section{Future Directions}

All but one study included in this review were performed using animals. Although these studies conducted on animals provide important information, it is unclear whether similar responses would be observed in humans. Further studies in humans poststroke are needed to identify the most appropriate protocol of treatment to increase BDNF concentration and its correlation with motor recovery. This is, however, complicated because it is unclear whether or not BDNF can cross the human bloodbrain barrier, which limits the interpretation of systemic BDNF measures in humans. In addition, more evidence is needed regarding systemic BDNF responses to exercise comparing the different phases of post-stroke recovery, such as acute, sub-acute and chronic phases.

\section{CONCLUSION}

The results of the current systematic review highlight that aerobic exercise can promote changes in central BDNF concentrations in animal models of stroke, while BDNF responses following non-aerobic exercises, such as reaching training and CIMT, are still controversial.

\section{REFERENCES}

1. Binder DK, Scharfman HE. Brain-derived neurotrophic factor. Growth Factors (2004) 22:123-31. doi: 10.1080/08977190410001723308

2. Baydyuk M, Xu B. BDNF signaling and survival of striatal neurons. Front. Cell. Neurosci. (2014) 8:254. doi: 10.3389/fncel.2014.00254

3. Schäbitz WR, Steigleder T, Cooper-Kuhn CM, Schwab S, Sommer C, Schneider A, et al. Intravenous brain-derived neurotrophic factor enhances poststroke sensorimotor recovery and stimulates neurogenesis. Stroke (2007) 38:2165-72. doi: 10.1161/STROKEAHA.106.477331

4. Ploughman M, Windle V, MacLellan CL, White N, Doré JJ, Corbett D. Brain-derived neurotrophic factor contributes to recovery of skilled reaching after focal ischemia in rats. Stroke (2009) 40:1490-5. doi: 10.1161/STROKEAHA.108.531806

5. Andreska T, Aufmkolk S, Sauer M, Blum R. High abundance of BDNF within glutamatergic presynapses of cultured hippocampal neurons. Front Cell Neurosci. (2014) 8:107. doi: 10.3389/fncel.2014.00107

6. Murer MG, Boissiere F, Yan Q, Hunot S, Villares J, Faucheux B, et al. An immunohistochemical study of the distribution of brainderived neurotrophic factor in the adult human brain, with particular reference to Alzheimer's disease. Neuroscience (1999) 88:1015-32. doi: 10.1016/S0306-4522(98)00219-X

7. Austin MW, Ploughman M, Glynn L, Corbett D. Aerobic exercise effects on neuroprotection and brain repair following stroke: a systematic review and perspective. Neurosci Res. (2014) 87:8-15. doi: 10.1016/j.neures.2014.06.007

8. Ploughman M, Austin MW, Glynn L, Corbett D. The effects of poststroke aerobic exercise on neuroplasticity: a systematic review of animal and clinical studies. Transl Stroke Res. (2015) 6:13-28. doi: 10.1007/s12975-014-0357-7

9. Kellner Y, Gödecke N, Dierkes T, Thieme N, Zagrebelsky M, Korte M. The BDNF effects on dendritic spines of mature hippocampal neurons depend on neuronal activity. Front Synapt Neurosci. (2014) 6:5. doi: 10.3389/fnsyn.2014.00005

10. Mang CS, Campbell KL, Ross CJ, Boyd LA. Promoting neuroplasticity for motor rehabilitation after stroke: considering the effects of aerobic exercise and genetic variation on brain-derived neurotrophic factor. Physical Therapy (2013) 93:1707-16. doi: 10.2522/ptj.20130053

11. Angevaren M, Aufdemkampe G, Verhaar HJ, Aleman A, Vanhees L. Physical activity and enhanced fitness to improve cognitive function in older people

\section{AUTHOR CONTRIBUTIONS}

CA, LG-S, MS-C, and GS conducted literature searches, selected the studies and wrote the manuscript. CA, LG-S, MS-C, GS, DR, and TR contributed to the editing of the manuscript, final version and approval.

\section{ACKNOWLEDGMENTS}

This study was supported by the São Paulo Research Foundation (FAPESP), Brazil (grants: \#2017/13655-6, \#2015/21695-2, \#2015/19197-4, \#2014/25845-6, and \#2013/ 25805-1).

\section{SUPPLEMENTARY MATERIAL}

The Supplementary Material for this article can be found online at: https://www.frontiersin.org/articles/10.3389/fneur. 2018.00637/full\#supplementary-material

Table 1S | Methodological quality of studies included in this review scored according to ARRIVE Guidelines.

without known cognitive impairment. Cochr Database Syst Rev. (2008) CD005381. doi: 10.1002/14651858.CD005381.pub3

12. Knaepen K, Goekint M, Heyman EM, Meeusen R. Neuroplasticity exercise-induced response of peripheral brain-derived neurotrophic factor: a systematic review of experimental studies in human subjects. Sports Med. (2010) 40:765-801. doi: 10.2165/11534530-000000000-00000

13. Dinoff A, Herrmann N, Swardfager W, Liu CS, Sherman C, Chan S, et al. The effect of exercise training on resting concentrations of peripheral brain-derived neurotrophic factor (BDNF): a meta-analysis. PLoS ONE (2016) 11:e0163037. doi: 10.1371/journal.pone.0163037

14. Kim MW, Bang MS, Han TR, Ko YJ, Yoon BW, Kim JH, et al. Exercise increased $\mathrm{BDNF}$ and trkB in the contralateral hemisphere of the ischemic rat brain. Brain Res. (2005) 1052:16-21. doi: 10.1016/j.brainres.2005.05.070

15. Zhang QW, Deng XX, Sun X, Xu JX, Sun FY. Exercise promotes axon regeneration of newborn striatonigral and corticonigral projection neurons in rats after ischemic stroke. PLoS ONE (2013) 8:e80139. doi: 10.1371/journal.pone.0080139

16. Banoujaafar H, Van Hoecke J, Mossiat CM, Marie C. Brain BDNF levels elevation induced by physical training is reduced after unilateral common carotid artery occlusion in rats. J Cereb Blood Flow Metab. (2014) 34:1681-7. doi: $10.1038 /$ jcbfm.2014.133

17. Ishida A, Misumi S, Ueda Y, Shimizu Y, Cha-Gyun J, Tamakoshi K, et al. Early constraint-induced movement therapy promotes functional recovery and neuronal plasticity in a subcortical hemorrhage model rat. Behav Brain Res. (2015) 284:158-66. doi: 10.1016/j.bbr.2015.02.022

18. Livingston-Thomas JM, Hume AW, Doucette TA, Tasker RA. A novel approach to induction and rehabilitation of deficits in forelimb function in a rat model of ischemic stroke. Acta Pharmacol Sin. (2013) 34:104-12. doi: 10.1038/aps.2012.106

19. El-Tamawy MS, Abd-Allah F, Ahmed SM, Darwish MH, Khalifa HA. Aerobic exercises enhance cognitive functions and brain derived neurotrophic factor in ischemic stroke patients. NeuroRehabilitation. (2014) 34:209-13. doi: 10.3233/NRE-131020

20. Maher CG, Sherrington C, Herbert RD, Moseley AM, Elkins M. Reliability of the PEDro scale for rating quality of randomized controlled trials. Phys Ther. (2003) 83:713-21. doi: 10.1093/ptj/83.8.713

21. Ploughman M, Granter-Button S, Chernenko G, Tucker BA, Mearow KM, Corbett D. Endurance exercise regimens induce differential effects 
on brain-derived neurotrophic factor, synapsin-I and insulin-like growth factor I after focal ischemia. Neuroscience (2005) 136:991-1001. doi: 10.1016/j.neuroscience.2005.08.037

22. Ploughman M, Granter-Button S, Chernenko G, Attwood Z, Tucker BA, Mearow KM, et al. Exercise intensity influences the temporal profile of growth factors involved in neuronal plasticity following focal ischemia. Brain Res. (2007) 1150:207-16. doi: 10.1016/j.brainres.2007.02.065

23. Ke Z, Yip SP, Li L, Zheng XX, Tong KY. The effects of voluntary, involuntary, and forced exercises on brain-derived neurotrophic factor and motor function recovery: a rat brain ischemia model. PLOS ONE (2011) 6:e16643. doi: 10.1371/journal.pone.0016643

24. Chen J, Qin J, Su Q, Liu Z, Yang J. Treadmill rehabilitation treatment enhanced BDNF-TrkB but not NGF-TrkA signaling in a mouse intracerebral hemorrhage model. Neurosci Lett. (2012) 529:28-32. doi: 10.1016/j.neulet.2012.09.021

25. Quirié A, Hervieu M, Garnier P, Demougeot C, Mossiat C, Bertrand $\mathrm{N}$, et al. Comparative effect of treadmill exercise on mature BDNF production in control versus stroke rats. PLOS ONE (2012) 7:e44218. doi: 10.1371/journal.pone.0044218

26. Lan X, Zhang M, Yang W, Zheng Z, Wu Y, Zeng Q, et al. Effect of treadmill exercise on 5-HT, 5-HT1A receptor and brain derived neurophic factor in rats after permanent middle cerebral artery occlusion. Neurol. Sci. (2014) 35:761-6. doi: 10.1007/s10072-013-1599-y

27. Seo TB, Kim TW, Shin MS, Ji ES, Cho HS, Lee JM, et al. Aerobic exercise alleviates ischemia-induced memory impairment by enhancing cell proliferation and suppressing neuronal apoptosis in hippocampus. Int. Neurourol. J. (2014) 18:187-97. doi: 10.5213/inj.2014.18.4.187

28. Sun J, Ke Z, Yip SP, Hu X, Zheng X, Tong K. Gradually increased training intensity benefits rehabilitation outcome after stroke by BDNF upregulation and stress suppression. Biomed Res Int. (2014) 2014:925762. doi: 10.1155/2014/925762

29. Ahn JH, Choi JH, Park JH, Kim IH, Cho JH, Lee JC, et al. Longterm exercise improves memory deficits via restoration of myelin and microvessel damage, and enhancement of neurogenesis in the aged gerbil hippocampus after ischemic stroke. Neurorehab Neural Repair. (2016) 30:894905. doi: 10.1177/1545968316638444

30. Himi N, Takahashi H, Okabe N, Nakamura E, Shiromoto T, Narita $\mathrm{K}$, et al. Exercise in the early stage after stroke enhances hippocampal brain-derived neurotrophic factor expression and memory function recovery. J Stroke Cerebrovasc Dis. (2016) 25:2987-94. doi: 10.1016/j.jstrokecerebrovasdis.2016.08.017

31. Takamatsu Y, Tamakoshi K, Waseda Y, Ishida K. Running exercise enhances motor functional recovery with inhibition of dendritic regression in the motor cortex after collagenase-induced intracerebral hemorrhage in rats. Behav Brain Res. (2016) 300:56-64. doi: 10.1016/j.bbr.2015.12.003

32. Yong MS, Hwangbo K. Skilled reach training influences brain recovery following intracerebral hemorrhage in rats. J Phys Ther Sci. (2014) 26:405-7. doi: $10.1589 /$ jpts. 26.405

33. Tamakoshi K, Kawanaka K, Onishi H, Takamatsu Y, Ishida K. Motor skills training improves sensorimotor dysfunction and increases microtubule-associated Protein 2 mRNA expression in rats with intracerebral hemorrhage. J Stroke Cerebrovasc Dis. (2016) 25:2071-7. doi: 10.1016/j.jstrokecerebrovasdis.2016.05.007

34. Ploughman M, Attwood Z, White N, Doré JJ, Corbett D. Endurance exercise facilitates relearning of forelimb motor skill after focal ischemia. Euro J Neurosci. (2007) 25:3453-60. doi: 10.1111/j.1460-9568.2007.05591.x
35. Yong MS, Kim SG, Cheon SH. Effects of skilled reach training with affected forelimb and treadmill exercise on the expression of neurotrophic factor following ischemia-induced brain injury in rats. J Phys Ther Sci. (2017) 29:647-650. doi: 10.1589/jpts.29.647

36. Hosp JA, Luft AR. Cortical plasticity during motor learning and recovery after ischemic stroke. Neural Plasticity (2011) 2011:871296. doi: $10.1155 / 2011 / 871296$

37. Holleran CL, Rodriguez KS, Echauz A, Leech KA, Hornby TG. Potential contributions of training intensity on locomotor performance in individuals with chronic stroke. J Neurol Phys Ther. (2015) 39:95-102. doi: 10.1097/NPT.0000000000000077

38. Leddy AL, Connolly M, Holleran CL, Hennessy PW, Woodward J, Arena RA, et al. Alterations in aerobic exercise performance and gait economy following high-intensity dynamic stepping training in persons with subacute stroke. $J$ Neurol Phys Ther. (2016) 40:239-48. doi: 10.1097/NPT.0000000000000147

39. Ivey FM, Stookey AD, Hafer-Macko CE, Ryan AS, Macko RF. Higher Treadmill Training Intensity to Address Functional Aerobic Impairment after Stroke. J Stroke Cerebrovasc Dis. (2015) 24:2539-46. doi: 10.1016/j.jstrokecerebrovasdis.2015.07.002

40. Coelho FG, Gobbi S, Andreatto CA, Corazza DI, Pedroso RV, Santos-Galduróz RF. Physical exercise modulates peripheral levels of brain-derived neurotrophic factor (BDNF): a systematic review of experimental studies in the elderly. Arch Gerontol Geriatr. (2013) 56:10-15. doi: 10.1016/j.archger.2012.06.003

41. Ferris LT, Williams JS, Shen CL. The effect of acute exercise on serum brainderived neurotrophic factor levels and cognitive function. Med Sci Sports Exerc. (2007) 39:728-34. doi: 10.1249/mss.0b013e31802f04c7

42. Huang T, Larsen KT, Ried-Larsen M, Møller NC, Andersen LB. The effects of physical activity and exercise on brain-derived neurotrophic factor in healthy humans: a review. Scand J Med Sci Sports (2014) 24:1-10. doi: 10.1111/sms.12069

43. Forti LN, Van Roie E, Njemini R, Coudyzer W, Beyer I, Delecluse C, et al. Dose-and gender-specific effects of resistance training on circulating levels of brain derived neurotrophic factor (BDNF) in community-dwelling older adults. Exp Gerontol. (2015) 70:144-9. doi: 10.1016/j.exger.2015.08.004

44. Goekint M, De Pauw K, Roelands B, Njemini R, Bautmans I, Mets T, et al. Strength training does not influence serum brain-derived neurotrophic factor. Euro J Appl Physiol. (2010) 110:285-93. doi: 10.1007/s00421-010-1461-3

45. Roig M, Skriver K, Lundbye-Jensen J, Kiens B, Nielsen JB. A single bout of exercise improves motor memory. PLoS ONE (2012) 7:e44594. doi: 10.1371/journal.pone.0044594

46. Quaney BM, Boyd LA, McDowd JM, Zahner LH, He J, Mayo MS, et al. Aerobic exercise improves cognition and motor function poststroke. Neurorehabit Neural Repair. (2009) 23:879-85. doi: 10.1177/1545968309338193

Conflict of Interest Statement: The authors declare that the research was conducted in the absence of any commercial or financial relationships that could be construed as a potential conflict of interest.

Copyright (c) 2018 Alcantara, García-Salazar, Silva-Couto, Santos, Reisman and Russo. This is an open-access article distributed under the terms of the Creative Commons Attribution License (CC BY). The use, distribution or reproduction in other forums is permitted, provided the original author(s) and the copyright owner(s) are credited and that the original publication in this journal is cited, in accordance with accepted academic practice. No use, distribution or reproduction is permitted which does not comply with these terms. 